\title{
Observable Quantities in Cosmological Models with Strings
}

\author{
Mariusz P. Dąbrowski and Jerzy Stelmach \\ Institute of Physics, University of Szczecin, Wielkopolska 15, 70-451 \\ Szczecin, Poland
}

\begin{abstract}
The Friedman equation for the universe with arbitrary curvature $(k=$ $0, \pm 1$ ), filled with mutually noninteracting pressureless dust, radiation, cosmological constant, and strings is considered. We assume the string domination scenario for the evolution of the latter component. Moreover, we discuss the simplest possibility for the scaling of the string energy density: $\varrho \propto R^{-2}$. For such models we write down the explicit solution of the Friedman equation. We realize that corresponding cosmological models do not essentially differ from those without strings. We find an analytic formula for the radial coordinate $\chi$ of a galaxy with a redshift $z$ and express it in terms of astronomical parameters. This relation is then used for the derivation of the astrophysical formulas such as luminosity distance, angular diameter, and source counts, which may serve for testing the string-dominated universe. It seems that the most sensitive test, at least from the formal point of view, is the formula for the number of galaxies $N(z)$ corresponding to a given value of the redshift. We show that the maximum of $N(z)$ strongly depends on the density of strings, especially if the density is large enough to explain the $\Omega$ problem. Other tests are also proposed.
\end{abstract}

\section{Subject headings: cosmology: observational}

Journal Reference: The Astronomical Journal 97, 978 (1989).

\section{Introduction}

One of the consequences of the inflationary scenario is the near flatness of the universe, which means that the energy density is very close to the critical value $\Omega=\varrho / \varrho_{c}=1$ (Guth 1981; for observational aspects, see also Loh 1986; Loh and Spillar 1986a,b). However, observation of galaxies gives us a value roughly one order 
less. A lack of observable mass necessary for approaching the critical density is usually called the dark matter problem. people propose many solutions to this problem (Turner 1987). One of the simplest solutons is the assumption that the cosmological constant does not vanish. Other possibilities are massive neutrinos, axions, or heavy superpartners of the usual particles, for example, photinos. Several years ago, when the theories of grand unification (GUTs) were used for the description of the early universe, one more candidate appeared to solve the mentioned problem - cosmic strings.

In gauge theories with spontaneous symmetry breaking, the phase transition at critical temperature (above which symmetry can be restored) can give rise to the nontrivial vacuum structure of the universe (Zel'dovich et al. 1974). These are domain walls, strings, or monopoles, depending on the topology of the manifold of degenerate vacua (Kibble 1976). More complicated topological objects such as walls bounded by strings or monopoles connected by strings can also be formed (Vachaspati and Vilenkin 1984). However, we have good reason to believe that, up to the present time, in our visible part of the universe only strings, or eventually an infinite network of strings with monopoles in vertices, could survive (Vilenkin 1981a; Vachaspati and Vilenkin 1987).

It is well-known that there are two extreme configurations to which a system of strings may evolve: a scaling configuration or a string-dominated universe (SDU). The former possibility has been extensively examined in the context of galaxy formation (Zel'dovich 1980; Vilenkin 1981b; Vilenkin and Shafi 1983; Turok and Brandenberger 1986; Kibble 1986). The latter one is also very attractive because it may give the explanation for the missing mass (Vilenkin 1984a; Turner 1985; Kibble 1986). We shall not discuss both scenarios, referring instead to the literature (Vilenkin 1985; Kibble 1985; Bennet 1986a,b; Scherer and Frieman 1986; Aryal et al. 1986). Our considerations are based on the result of Turok and Bhattacharjee (1984), who have shown that, neglecting interaction, the energy density of a network of strings scales as $\varrho_{s} \propto R^{-n}$, where $2 \leq n \leq 3$ (see also Kibble (1986) and Vachaspati and Vilenkin (1987). The case $n=2$ and the astronomical constraints on SDU have been examined by Gott and Rees (1987). Earlier, Gott (1985) had given exact solution and presented interesting considerations concerning the gravitational field of strings in the context of gravitational lensing (see also Hiscock (1985)).

The purpose of the present paper is to calculate some atrophysical formulas for the homogeneous and isotropic universe with arbitrary curvature $(k=0, \pm 1)$ filed with pressureless dust, radiation, cosmological constant, and the system of strings. For simplicity, we consider only an extreme case when $n=2$ (it corresponds, for 
example, to the set of randomly oriented straight strings or to the tangled network of strings which conformally stretches by the expansion). This case is particularly interesting because it allows treatment of all mentioned components of the universe simultaneously in an analytic way. Generally, this is not the case if $2 \leq n \leq 3$. Although the case under consideration is probably too idealistic, it gives some aspects of observational problems in the universe with strings and in some sense completes the discussion of the string-dominated universe given by Vilenkin, Kibble, and Kardashev (1986), Charlton and Turner (1987), or Gott and Rees (1987).

Ther exist several observational tests of the cosmic-string scenario: gravitational lensing (Vilenkin 1984b), anisotropy in the microwave-background radiation (Kaiser and Stebbins 1984), and gravitational radiation emitted by decaying loops (Hogan and Rees 1984). The tests proposed in the present paper are of a different type and are not relevant for detecting a single string. Our tests could be applied only for the universe in which the network of strings is sufficiently dense in order to influence astrophysical observables such as luminosity distance, angular diameter, or source counts. Moreover, the strings under consideration should be relatively light (Vilenkin 1984a).

We do not want to make a statement pro or con regarding the string scenario. Our task is to propose tests that could be used for the verification of the hypothesis concerning a universe dominated by a network of strings. It seems to us that at least one of them, namely source counts, is very promising for this purpose. We leave consideration of the less trivial case of the string-domination scenario (where $3>n>2$ and the universe behaves very like a matter-dominated one) for the future.

In the next section, we introduce some definitions and we describe the Friedmann equation for the universe with strings in a form that allows use of the methods and results (with some modifications) from our previous papers concerning the case without strings (Dạbrowski and Stelmach 1986a,b; 1987a). Next, we give analytic solutions of the Friedman equation in terms of Weierstrass elliptic and nonelliptic functions. In Sec. III, we find the relations between astronomical parameters and derive some astrophysical formulas for the universe with strings. Section IV is devoted to the discussion of the tests for the existence of the network of cosmic strings. We pay much attention to the case where the formulas are given by elementary functions. Because of their simplicity, these cases are of special interest. In Sec. V we summarize the results. 


\section{Friedman Equation Including Strings}

We consider Friedman models described by the equation

$$
\dot{R}^{2}+k=\frac{8 \pi G}{3} \varrho R^{2}
$$

where $\varrho$ is total energy density of the universe including, besides the usual components $\varrho_{m}$ - matter, $\varrho$ - radiation, $\varrho_{\Lambda}-$ cosmological constant), also strings $\varrho_{s}$ :

$$
\varrho=\varrho_{r}+\varrho_{m}+\varrho_{s}+\varrho_{\Lambda}
$$

$k=0, \pm 1$ is the curvature index and $R(t)$ is the scale factor. We assume that strings satisfy the equation of state (Zel'dovich 1980, Vilenkin 1981c)

$$
p_{s}=-\frac{\varrho_{s}}{3} \text {. }
$$

Noninteraction between the components gives the simple relation for $\varrho_{s}$, namely

$$
\varrho_{s} R^{2}=C_{s} \frac{3}{8 \pi G} .
$$

Following the notation of Coquereaux and Grossmann (1982), we give the similar expressions for $\varrho_{r}, \varrho_{m}$, and $\varrho_{\Lambda}$ :

$$
\begin{aligned}
\varrho_{r} R^{4} & =C_{r} \frac{3}{8 \pi G}, \\
\varrho_{m} R^{3} & =C_{m} \frac{3}{8 \pi G}, \\
\varrho_{\Lambda} & =\frac{3}{8 \pi G},
\end{aligned}
$$

where $C_{r}, C_{m}$, and $C_{s}$ are constants. If we now introduce a new parameter

$$
k^{\prime} \equiv k-C_{s}
$$

Friedmann equation (1) acquires a form identical to that without strings with the only replacement $k \rightarrow k^{\prime}$

$$
\dot{R}^{2}+k=\frac{C_{m}}{R}+\frac{C_{r}}{R^{2}}+\frac{\Lambda}{3} R^{2} .
$$

However, generally the sign of $k^{\prime}$ now has nothing to do with the curvature of the universe.

The formal resemblance of both models (with and without strings) notably simplifies treatment of the present case. In deriving different expressions for the models 
without strings (Dąbrowski and Stelmach 1986a,b; 1987b), for the most part we did not employ the exact value of $k$. Thus, almost all formulas are still valid if we put $k^{\prime}$ instead of $k$. For example, Eq. (9) rewritten in terms of dimensionless variables and parameters takes the form

$$
\left(\frac{d T}{d \tau}\right)^{2}=\alpha T^{4}+\frac{2}{3} T^{3}-k^{\prime} T^{2}+\frac{\lambda}{3}
$$

where

$$
\begin{aligned}
\lambda & =\frac{\Lambda}{\Lambda_{c}}, \\
\Lambda_{c} & =\frac{4}{\left(9 C_{m}^{2}\right)}, \\
\alpha & =C_{r} \Lambda_{c}, \\
d \tau & =\frac{d t}{R}, \\
T(\tau) & =\Lambda_{c}^{-\frac{1}{2}} R^{-1}(\tau) .
\end{aligned}
$$

The solution of the Friedman equation may now be written down in a parametric representation in at least two ways. In the first version, we employ the Weierstrass elliptic function $\mathcal{P}$, given as the solution of a differential equation (Tricomi 1937; Abramovitz and Stegun 1964)

$$
\left(\frac{d \mathcal{P}}{d \tau}\right)^{2}=4 \mathcal{P}^{3}-g_{2} \mathcal{P}-g_{3}
$$

where

$$
g_{2}=\frac{k^{\prime 2}}{12}+\frac{\alpha \lambda}{3}
$$

and

$$
g_{3}=6^{-3}\left(k^{\prime 3}-2 \lambda\right)-\frac{\alpha \lambda k^{\prime}}{18}
$$

Then, the solution reads

$$
R(\tau)=\frac{1}{6 \sqrt{\Lambda_{c}}} \frac{3 \sqrt{\alpha} \mathcal{P}^{\prime}(\tau)+\mathcal{P}(\tau)+\frac{k^{\prime}}{12}}{\left[\mathcal{P}(\tau)+\frac{k^{\prime}}{12}\right]^{2}-\frac{\alpha \lambda}{12}}
$$

In the second case, nonelliptic functions $\zeta$ and $\sigma$ are used:

$$
R(\tau)=\sqrt{\frac{3}{\Lambda}}\left[\zeta\left(\tau-\tau_{g}\right)-\zeta\left(\tau+\tau_{f}\right)+\zeta\left(\tau_{g}\right)-\zeta\left(\tau_{f}\right)\right]
$$




$$
\left(\frac{\Lambda}{3}\right)^{\frac{1}{2}} t(\tau)=\tau\left[\zeta\left(\tau_{g}\right)-\zeta\left(\tau_{f}\right)\right]+\ln \left|\frac{\sigma\left(\tau-\tau_{g}\right) \sigma\left(\tau_{f}\right)}{\sigma\left(\tau-\tau_{f}\right) \sigma\left(\tau_{g}\right)}\right| .
$$

The numbers $\tau_{f}$ and $\tau_{g}$, which are generally complex, are given by the formulas (Da̧browski and Stelmach 1987a)

$$
\begin{aligned}
& \tau_{f}=\mathcal{P}^{-1}\left(-\frac{k^{\prime}}{12}-\frac{1}{2} \sqrt{\frac{\alpha \lambda}{3}}\right), \\
& \tau_{f}=\mathcal{P}^{-1}\left(-\frac{k^{\prime}}{12}+\frac{1}{2} \sqrt{\frac{\alpha \lambda}{3}}\right) .
\end{aligned}
$$

The discussion of the solution proceeds analogously as in the case without strings (Coquereaux and Grossmann 1982; Dąbrowski and Stelmach 1986a). For details, we refer to Dąbrowski and Stelmach (1987b). We realize that, in general, the types of the solutions do not essentially differ from the case without strings (when $k^{\prime}=k=0, \pm 1$ ). The fundamental formal difference is that for the universe with strings $k^{\prime}$ is not normalized and may take arbitrary values from the interval $(-\infty, 1>$. However, it should be stresses that some connection between $k$ and $k^{\prime}$ exists and follows from Eq. (8). Namely, for $k^{\prime}>0$, there is only one possibility, $k=1$, for $k^{\prime}=0$ the universe may be closed $(k=1)$ or flat $(k=0)$, and finally for $k^{\prime}<0$ three possibilities may occur $(k=0, \pm 1)$.

At the end of this section we discuss some solutions that take on an especially simple form. These are the cases when the Friedman equation is explicitly integrable, i.e., the Weierstrass functions $\mathcal{P}, \zeta$, and $\sigma$ degenerate to elementary ones. We shall not find all such solutions, but, because of their particular simplicity, models with a vanishing cosmological constant $(\lambda=0)$ are of special interest. We get oscillating models for $k^{\prime}>0$ :

$$
\begin{aligned}
R(\tau) & =\frac{1}{3 k^{\prime} \sqrt{\Lambda_{c}}}\left(1-\cos \sqrt{k^{\prime}} \tau+3 \sqrt{\alpha k^{\prime}} \sin \sqrt{k^{\prime}} \tau\right), \\
t(\tau) & =\frac{1}{3 k^{\prime} \sqrt{\Lambda_{c}}}\left[\tau-\frac{1}{\sqrt{k^{\prime}}} \sin \sqrt{k^{\prime}} \tau-3 \sqrt{\alpha}\left(\cos \sqrt{k^{\prime}} \tau-1\right)\right],
\end{aligned}
$$

and monotonic ones for $k^{\prime}<0$,

$$
\begin{aligned}
R(\tau) & =\frac{1}{3 k^{\prime} \sqrt{\Lambda_{c}}}\left(1-\cosh \sqrt{-k^{\prime}} \tau+3 \sqrt{-\alpha k^{\prime}} \sinh \sqrt{-k^{\prime}} \tau\right), \\
t(\tau) & =\frac{1}{3 k^{\prime} \sqrt{\Lambda_{c}}}\left[\tau-\frac{1}{\sqrt{-k^{\prime}}} \sinh \sqrt{-k^{\prime}} \tau-3 \sqrt{\alpha}\left(\cosh \sqrt{-k^{\prime}} \tau-1\right)\right],
\end{aligned}
$$

and for $k^{\prime}=0$,

$$
R(\tau)=\frac{1}{6 \sqrt{\Lambda_{c}}} \tau(\tau+6 \sqrt{\alpha})
$$




$$
t(\tau)=\frac{1}{2 \sqrt{\Lambda_{c}}} \tau^{2}\left(\frac{1}{9} \tau+\sqrt{\alpha}\right) .
$$

The last two formulas, which describe either the flat universe without strings $(k=$ $\left.0, C_{s}=0\right)$ or the closed universe with strings $\left(k=1=C_{s}\right)$, follow from Eqs. (24) and (26) by taking the limit $k^{\prime} \rightarrow 0$. These are the cases in which radiation and matter are negligible $\left(C_{r}=C_{m}=0\right)$, which can happen in a vacuum or in a string-dominated universe. Explicit integration of Eq. (9) gives for different values of $k^{\prime}$ and $\Lambda$ solutions that are qualitatively the same as in the stringless cosmology:

$$
\begin{aligned}
& R(t)=\sqrt{3 k^{\prime} / \Lambda} \cosh \sqrt{\Lambda / 3} t, \text { for } k^{\prime}, \Lambda>0, \\
& R(t)=\sqrt{-3 k^{\prime} / \Lambda} \sinh \sqrt{\Lambda / 3} t, \text { for } k^{\prime}<0, \Lambda>0, \\
& R(t)=\exp \sqrt{\Lambda / 3} t, \text { for } k^{\prime}=0, \Lambda>0, \\
& R(t)=\sqrt{3 k^{\prime} / \Lambda} \sinh \sqrt{-\Lambda / 3} t, \text { for } \Lambda, k^{\prime}>0,
\end{aligned}
$$

and, finally,

$$
R(t)=\sqrt{-k^{\prime}} t, \text { for } \Lambda=0, k^{\prime}<0 .
$$

For simplicity, we put integration constants equal to zero. Note that the last formula describes also the asymptotic behaviour of the monotonic model given by eqs. (26). Some numerical calculation for the above models, especially for that given by Eq. (33), were performed by Kardashev (1986).

\section{Astrophysical Formulas}

Detailed discussion of the relations between observable quantities in the usual Friedmann models has been performed in our recent paper (Da̧browski and Stelmach 1987a). It has been pointed out in the present work that the extension to the case with cosmic strings may be easily achieved by formal replacement $k \rightarrow k^{\prime}$. Definitive solution of the problem consists therefore in expressing $k^{\prime}$ in terms of astronomical parameters $q_{0}, \sigma_{r 0}, \sigma_{m 0}, \sigma_{s 0}$. The last parameter did not come out so far in our papers. Its appearance follows from the existence of cosmic strings in the model and it is defined

$$
\sigma_{s 0}=\frac{4 \pi G \varrho_{s 0}}{3 H_{0}^{2}}
$$

where $H_{0}$ and $\varrho_{s 0}$ are present values of the Hubble constant and the energy density of strings, respectively. We calculate $k^{\prime}$ using the definition

$$
k^{\prime}=k-C_{s}=k-2 \sigma_{s 0} H_{0}^{2} R_{0}^{2}
$$


and next ruling out $H_{0}^{2} R_{0}^{2}$ from the relation

$$
H_{0}^{2} R_{0}^{2}=\frac{k^{\prime}}{4 \sigma_{r 0}+3 \sigma_{m 0}-q_{0}-1} .
$$

Finally, $k^{\prime}$ reads

$$
k^{\prime}=\left(4 \sigma_{r 0}+3 \sigma_{m 0}-q_{0}-1\right) \frac{k}{4 \sigma_{r 0}+3 \sigma_{m 0}+2 \sigma_{s 0}-q_{0}-1} .
$$

Remaining parameters determining the model expressed in terms of $\sigma_{r 0}, \sigma_{m 0}, \sigma_{s 0}, H_{0}$, and $q_{0}$ are

$$
\begin{aligned}
\Lambda_{c} & =\frac{H_{0}^{2}}{9 \sigma_{m 0}^{2}}\left(\frac{4 \sigma_{r 0}+3 \sigma_{m 0}-q_{0}-1}{k^{\prime}}\right)^{3}, \\
\Lambda & =3 H_{0}^{2}\left(2 \sigma_{r 0}+\sigma_{m 0}-q_{0}\right), \\
\lambda & =27 \sigma_{m 0}^{2}\left(2 \sigma_{r 0}+\sigma_{m 0}-q_{0}\right)\left(\frac{k^{\prime}}{4 \sigma_{r 0}+3 \sigma_{m 0}-q_{0}-1}\right)^{3}, \\
\alpha & =\frac{2 \sigma_{r 0}}{9 \sigma_{m 0}^{2}} \frac{4 \sigma_{r 0}+3 \sigma_{m 0}-q_{0}-1}{k^{\prime}} .
\end{aligned}
$$

For completeness, we define $\sigma_{r 0}, \sigma_{m 0}, H_{0}$, and $q_{0}$ :

$$
\begin{aligned}
\sigma_{r 0} & =\frac{4 \pi G \varrho_{r 0}}{3 H_{0}^{2}}, \\
\sigma_{m 0} & =\frac{4 \pi G \varrho_{m 0}}{3 H_{0}^{2}} \\
H_{0} & =\frac{\dot{R}}{R} \\
q_{0} & =-\frac{\ddot{R}_{0} R_{0}}{\dot{R}_{0}^{2}}
\end{aligned}
$$

where a zero means that the magnitudes correspond to the present value of the cosmic time $t_{0}$. We note that application of the formula (38) to the expression (39), (41), and (42) removes the explicit dependence on $k^{\prime}$, however, at the cost of also removing the proper parameter describing strings $\sigma_{s 0}$. From the same formula,together with Eq. (37), a very interesting property of the models with strings may be deduced, namely, changing of the curvature of the universe without altering its dynamics (Gott and Rees 1987). Gott and Rees come to this conclusion by investigating the local influence of the strings on the geometry of the universe. In our approach, the system of strings forms a continuous fluid satisfying the exotic equation of state $p=-\frac{1}{3} \varrho$. In this sense, we examine the global influence of strings on the evolution. In order to see how the 
above property follows from our model, let us come back to the formula (38). Let us assume for the moment that strings are absent $\left(\sigma_{s 0}=0\right)$ and, for example $k=0$. Then, from the construction $4 \sigma_{r 0}+3 \sigma_{m 0}-q_{0}-1$ and $k^{\prime}=0$. Next, we add strings $\left(\sigma_{s 0}>0\right)$, leaving other astronomical parameters $\left(\sigma_{r 0}, \sigma_{m 0}, q_{0}\right)$ unchanged. Then, of course $4 \sigma_{r 0}+3 \sigma_{m 0} 2 \sigma_{s 0}-q_{0}-1>0$ and consequently, the curvature index $k$ has to be equal to 1 . However, $4 \sigma_{r 0}+3 \sigma_{m 0}-q_{0}-1$ is still zero. Hence, $k^{\prime}=0$ and the dynamics of the universe, which depends on the sign of $k^{\prime}$, remains the same, although the curvature changed. The same conclusion may also be deduced if $k= \pm 1$ from the beginning. It seems that the reason for this property is that the dynamics of the universe depends in principle (besides the $\Lambda$ term) on the sign of the expression $4 \sigma_{r 0}+3 \sigma_{m 0}-q_{0}-1$, which does not include strings (see Eq. (37)). For completeness, it has to be stressed that adding an exotic fluid other than the above one (for example, domain walls) changes the dynamics because of the appearance of a qualitatively new term on the right-hand side of Eq. (9).

Now we proceed to the presentation of the astrophysical formulas for the universe with strings. A magnitude that enters most of the expressions is a redshift $z$ of observed galaxies. We start with the fundamental relation that establishes the connection between the redshift and a radial coordinate $\chi$ of a galaxy. In the most general case with cosmic strings, radiation, and a cosmological term this relation is nontrivial and the Weierstrass elliptic function $\mathcal{P}$ is used

$$
\begin{aligned}
\mathcal{P}(\chi) & =\frac{k}{4 \sigma_{r 0}+3 \sigma_{m 0}+2 \sigma_{s 0}-q_{0}-1}\left(\frac{4 \sigma_{r 0}+3 \sigma_{m 0}-q_{0}-1}{6}-\frac{z+2}{2}\left[\sigma_{m 0}+\sigma_{r 0}(z+2)\right]\right. \\
& \left.+\frac{1}{4 z^{2}}\left\{1+\left[2 \sigma_{r 0} z^{2}(z+2)^{2}+\sigma_{m 0} z^{2}(2 z+3)+q_{0} z(z+2)+(z+1)^{2}\right]^{\frac{1}{2}}\right\}\right) .
\end{aligned}
$$

The $\chi$ coordinate, by definition, is a difference between the present value of the conformal time $\tau_{0}$ and a time $\tau$ corresponding to the moment of the emission of the light ray by the galaxy with the redshift $z$ :

$$
\chi=\tau_{0}-\tau .
$$

If we compare the above formula with the appropriate one in the model without strings (Da̧browski and Stelmach 1986b, 1987a), we perceive that the difference is quite unremarkable. As a matter of fact, it is no wonder, otherwise cosmic strings would have been already discovered. Nevertheless, the distinction exists and should come out in almost every astrophysical formula. Following our last paper (Dąbrowski and Stelmach 1987a), we specify some expressions emphasizing particularly the universe with vanishing a $\Lambda$ term and those that are string dominated.

\section{a) The Luminosity Distance}


Denoting the right-hand side of Eq. (47) by $f(z)$, the formula for the luminosity distance of the observed galaxy is

$$
D_{0}=\frac{z+1}{H_{0}}\left(\frac{k}{4 \sigma_{r 0}+3 \sigma_{m 0}+2 \sigma_{s 0}-q_{0}-1}\right)^{\frac{1}{2}} S_{k}(\chi),
$$

where

$$
\chi=\mathcal{P}^{-1}[f(z)]
$$

and $S_{k}$ is defined

$$
S_{k}(\chi)=\left\{\begin{array}{l}
\sin \chi \quad k>0 \\
\chi \quad k=0 \\
\sinh \chi \quad k<0
\end{array}\right.
$$

In degenerate cases $(\lambda=0)$ the Weierstrass $\mathcal{P}$ function converts into an elementary one (Abramovitz and Stegun 1964):

$$
\mathcal{P}(\chi)=-\frac{k^{\prime}}{12}+\frac{\left|k^{\prime}\right|}{4 S_{k^{\prime}}^{2}\left(\frac{\left|k^{\prime}\right|^{\frac{1}{2}}}{2} \chi\right)} .
$$

Then, the formula for $\chi$ reads

$$
\begin{aligned}
\chi & =\frac{1}{\left|k^{\prime}\right|^{\frac{1}{2}}} S_{k^{\prime}}^{-1}\left[\frac{\left|k^{\prime}\right|^{\frac{1}{2}}}{z+1}\left(\frac{2 \sigma_{r 0}+2 \sigma_{m 0}+2 \sigma_{s 0}-1}{k}\right)^{\frac{1}{2}}\right. \\
& \left.\times \frac{\sigma_{m 0} z+\left(\sigma_{m 0}+2 \sigma_{r 0}-1\right)\left(\left[2 \sigma_{r 0} z(z+2)+2 \sigma_{m 0} z+1\right]^{\frac{1}{2}}-1\right)}{\sigma_{m 0}^{2}+2 \sigma_{r 0}\left(2 \sigma_{r 0}+2 \sigma_{m 0}-1\right)}\right] .
\end{aligned}
$$

In models without strings $k^{\prime}=k=0, \pm 1$, in consequence we have

$$
\frac{1}{\left|k^{\prime}\right|^{\frac{1}{2}}} S_{k^{\prime}}^{-1}\left(\left|k^{\prime}\right|^{\frac{1}{2}} \ldots\right)=S_{k}^{-1}(\ldots) \text {. }
$$

Inserting Eq. (53) into (49) and remembering that removing $\lambda$ allows elimination of $q_{0}$ (cf. Eq. (41), we get the formula for the luminosity distance in a form that does not depend explicitly on curvature. In the universe with strings, this is not the case. parameters $k^{\prime}$ and $k$ generally do not coincide. Hence the composition $S_{k}\left(\left|k^{\prime}\right|^{-\frac{1}{2}} S_{k^{\prime}}^{-1}\right)$ does not yield the identical function and the redshift-magnitude formula has a more complicated form. All formulas significantly simplify only in extreme cases. For example, choosing $\sigma_{r 0}=0, \sigma_{m 0}=\sigma_{s 0}=1 / 2$ and $k=1$, we have

$$
\chi=2\left(1-\frac{1}{\sqrt{z+1}}\right)
$$


which coincides with the result known from the stringless cosmology. Of course, this similarity is not accidental. The reason is that in the case considered here the parameter $k^{\prime}$, which is some sense plays the role of the curvature index, is equal to zero. Hence some cosmological effects following from the existence of cosmic strings compensate for those typical for closed geometry $(k=1)$. In spite of this, the formulas for the luminosity distance are in both cases different (especially for large $z$ )

$$
\begin{aligned}
& D_{0}=\frac{z+1}{H_{0}} \sin \left[2\left(1-\frac{1}{\sqrt{z+1}}\right)\right], \text { for } k=1, \sigma_{s 0}=\frac{1}{2}, \\
& D_{0}=\frac{z+1}{H_{0}} 2\left(1-\frac{1}{\sqrt{z+1}}\right), \text { for } k=0=\sigma_{s 0}=0 .
\end{aligned}
$$

Another interesting case may be obtained when $\sigma_{s 0}$ is arbitrary but radiation and matter are negligible compared to the density of strings. Then, the expression for $D_{0}$ is

$$
D_{0}=\frac{z+1}{H_{0}}\left(\frac{k}{2 \sigma_{s 0}-1}\right)^{\frac{1}{2}} S_{k}\left[\left(\frac{2 \sigma_{s 0}-1}{k}\right)^{\frac{1}{2}} \ln (z+1)\right]
$$

and simplifies further by putting $k=0$

$$
D_{0}=\frac{z+1}{H_{0}} \ln (z+1)
$$

Note that adding the cosmological constant to the case $\left(\sigma_{r 0}=\sigma_{m 0}=0\right)$ does not lead beyond elementary functions (cf. Eqs. (30)-(34)). In order to find $\chi$, we use the formulas

$$
\begin{aligned}
\chi & =\tau_{0}-\tau=\int_{\tau}^{\tau_{0}} d \tau=\int_{t}^{t_{0}} \frac{d t}{R(t)}, \\
\frac{R_{0}}{R(t)} & =z+1 \\
R_{0} & =\frac{1}{H_{0}}\left(\frac{k}{2 \sigma_{s 0}-q_{0}-1}\right)^{\frac{1}{2}} \\
k^{\prime} & =-\left(q_{0}+1\right) \frac{k}{2 \sigma_{s 0}-q_{0}-1}, \\
\Lambda & =-3 q_{0} H_{0}^{2},
\end{aligned}
$$

where $R(t)$ is given by Eqs.(30)-(34). performing simple integrations, we get

$$
\chi=\sqrt{2 \sigma_{s 0}} z \text { for } k^{\prime}=0
$$

which implies that $k=1, q_{0}=-1, \Lambda=3 H_{0}^{2}$,

$$
\chi=\left(\frac{1+q_{0}-2 \sigma_{s 0}}{q_{0}+1}\right)^{\frac{1}{2}}\left\{\arctan \left(\frac{-1}{q_{0}+1}\right)^{\frac{1}{2}}-\arctan \left[\frac{q_{0}}{\left(q_{0}+1\right)(z+1)^{2}}-1\right]^{\frac{1}{2}}\right\} \text { for } k^{\prime}>0,
$$


which implies that $k=1, q_{0}<-1, \Lambda>3 H_{0}^{2}$, and

$$
\chi=\left(\frac{1+q_{0}-2 \sigma_{s 0}}{q_{0}+1}\right)^{\frac{1}{2}} \ln \frac{(z+1)\left(q_{0}+1\right)^{\frac{1}{2}}+\left[\left(q_{0}+1\right)(z+1)^{2}-q_{0}\right]^{\frac{1}{2}}}{\left(q_{0}+1\right)^{\frac{1}{2}}+1} \text { for } k^{\prime}<0,
$$

which implies that $k=0, \pm 1, q_{0}>-1, \Lambda<3 H_{0}^{2}$. The case where the $\Lambda$ term vanishes can be recovered only from the last formula (66) by putting $q_{0}=0$ (see Eq. (63)).

\section{b) Angular Diameter}

Writing an expression for the angular diameter $\theta$ of a galaxy with the redshift $z$ in the universe with strings does not lead to any difficulaties. We just use the known formula

$$
\theta=\frac{d(z+1)}{D_{0}}
$$

where $d$ is the linear size of the galaxy and $D_{0}$ its luminosity distance given by Eq. (49). In this context, one usually discusses minimal values of $\theta$, since it turns out taht in the expanding universe the angular diameter of a galaxy is not a decreasing function of its redshift. In the next section, we shaw that $\theta_{\text {min }}$ depends distinctly on the density of strings. Therefore, observation of $\theta_{\min }$ may be a good tool for testing a string-dominated universe.

\section{c) Source Counts}

We calculate the number of sources with redshifts from the interval $z, z+d z$. We use the same formula as in the standard cosmology without strings,

$$
d N=4 \pi n(z) S_{k}^{2}[\chi(z)] \frac{d \chi}{d z} d z
$$

however, the $\chi$ coordinate is now given by Eq. (47). Such a general formula, although exact, is troublesome in practical applications because its complicated form. Of course, in some special cases the expression simplifies a little bit (cf. Eqs. (53) and (64)-(66), see also Eq.(46) from our previous paper (Da̧browski and Stelmach 1987a). However, notable amplification is obtained only by expanding the appropriate expressions into series with respect to $z$. We find that source counts are the strongest test for the cosmic strings hypothesis. We shall discuss this point in detail in the next section.

There exist some other cosmological formulas that may be found in the models under considerations: particle and event horizon, the age, or eventually maximum 
radius and period of oscillation of the universe. In the quoted preprint (Dạbrowski and Stelmach 1987b), we have derived these magnitudes and we discussed in detail the influence of cosmic strings on them. In the present paper, we do not want to pay too much attention to this. The task of the first-rate significance is the verification of the string-domination scenario on the basis of the strongest tests.

\section{Astrophysical Tests for the Cosmic Strings}

Writing down the solutions of the Friedmann equation in Sec. II we realized that resulting cosmological models including strings should be very similar to those without strings. We concluded that the cosmic string network should be hardly detectable. To be more precise, we consider now the formula for the luminosity distance $D_{0}$ to a galaxy with a redshift $z$. The complicated form of the general formula (49) renders difficulties while fitting it to observational data. Because of this and also because of the small accuracy of the data it seems that using approximate expressions for the relation is justified. We perform an expansion of $D_{0}$ in the general case with radiation, matter, cosmological constant, and strings into series with respect to the redshift. The resulting expression is (see also Solheim 1966)

$$
D_{0}=\frac{1}{H_{0}}\left\{z+\frac{1}{2}\left(1-q_{0}\right) z^{2}+\frac{1}{6}\left[3 q_{0}\left(q_{0}+1\right)-12 \sigma_{r 0}-6 \sigma_{m 0}-2 \sigma_{s 0}\right] z^{3}+\ldots\right\} .
$$

Note that $D_{0}$ is the luminosity distance, not a corrected one as in the paper of Ellis and Maccallum (1970). The appearance of $\sigma_{s 0}$ not before the third order of the expansion means that the luminosity-distance relation is not a good test for the existence of strings.

As a matter of fact, in astrophysical tests, instead of the luminosity distance $D_{0}$ one rather measures the apparent magnitude $m$ given by the formula (Lang 1974)

$$
m=5 \log D_{0}+M+\text { const. }
$$

where $M$ is the absolute magnitude. Expansion of the magnitude (in the corrected form) into series (cf. Kristian and Sachs 1966, Ellis and MacCallum 1970) gives

$$
\begin{aligned}
m & =M-5 \log _{10} H_{0}+5 \log z \\
& +\left(2.5 \log _{10} e\right)\left\{\left(1-q_{0}\right) z+\left[\frac{1}{4}\left(3 q_{0}+1\right)\left(q_{0}-1\right)-\frac{2 \sigma_{s 0}}{3}-\frac{2 \Lambda_{(4)}}{3 H_{0}^{2}}\right] z^{2}+\ldots\right\} .
\end{aligned}
$$

Here $\Lambda$ is given by Eq. (40). Note that the radiation is excluded in the above formula by means of $\Lambda$. here we notice that the strings appear in the second order. It seems 
that the influence of strings is still weak. From the point of view of the possibility of the detection of the cosmic strings, this result is not promising.

Now we consider another test connected with the minimal angular diameter of galaxies. The value of the redshift $z_{\min }$ that corresponds to $\theta_{\min }$ has been calculated analytically for pressureless Friedman models without the $\Lambda$ term and strings (Edwards 1972, narlikar 1983), $z_{\min }=\frac{5}{4}$. We showed in our recent paper (Da̧browski and Stelmach 1987a) that adding the radiation in a flat universe lowers this value:

$$
z_{\text {min }}=\frac{1}{4 \sigma_{r 0}}\left(2 \sigma_{r 0}-1+\sqrt{6 \sigma_{r 0}}\right) \leq \frac{5}{4} .
$$

Calculation of $z_{\min }$ in a general case, especially including strings, is complicated because it leads to a non-elementary equation,

$$
\frac{S_{k}(\chi)}{S_{k}^{\prime}(\chi)}=(z+1) \frac{d \chi}{d z}
$$

It seems that an analytic solution exists only in one special case when $k=\lambda=\sigma_{r 0}=$ $\sigma_{m 0}=0$, i.e., in a string-dominated universe. Then

$$
\chi=\ln (z+1)
$$

and Eq. (73) gives

$$
z_{\text {min }}=e-1 \text {. }
$$

In cosmological scale, the calculated value is remarkably greater than the value $\frac{5}{4}$ obtained in a matter-dominated universe. In order to see how $z_{\min }$ changes if the universe passes from the matter-dominated state to the string-dominated one, a nonelementary equation has to be solved. For example, in the case $k=\lambda=\sigma_{r 0}=0$ (but $\sigma_{m 0} \neq 0$ and $\sigma_{s 0} \neq 0$ ), Eq. (73) takes the form ( $\chi$ is given by Eq. (53))

$$
\begin{aligned}
\sinh \sqrt{\frac{2 \sigma_{s 0}}{z\left(1-2 \sigma_{s 0}\right)+1}} & =\frac{\sqrt{2 \sigma_{s 0}}}{\left(\frac{1}{2}-\sigma_{s 0}\right)^{2}} \\
& \times \frac{z\left(\frac{1}{2}-\sigma_{s 0}\right)-\left(\sigma_{s 0}+\frac{1}{2}\right)\left(\sqrt{z\left(1-2 \sigma_{s 0}\right)+1}-1\right)}{z+1} .
\end{aligned}
$$

Its numerical solution with respect to $z$, for any value of $\sigma_{s 0} \in\left(0, \frac{1}{2}\right)$ is presented in the form of a diagram (1).

The influence of the $\Lambda$ term on $z_{\text {min }}$ may also be examined. We perform it for the string-dominated model. Then $\chi$ is given by Eq. (66), and the equation to be solved is

$$
\ln \frac{z+1+\left[(z+1)^{2}-q_{0} /\left(q_{0}+1\right)\right]^{\frac{1}{2}}}{1+1 /\left(q_{0}+1\right)}=\frac{z+1}{\left[(z+1)^{2}-q_{0} /\left(q_{0}+1\right)\right]^{\frac{1}{2}}} .
$$


In Fig. 2 we illustrate the dependence of $z_{\min }$ on the deceleration parameter, which is proportional to the cosmological constant in the case considered (cf. Eq. (63)). Since the signs of the $\Lambda$ term and $q_{0}$ are opposite, we deduce from the diagram that the negative cosmological constant, even when large, insignificantly lowers the value of the redshift $z_{\text {min }}$, for which the engular size of a galaxy is minimal, while the positive $\Lambda$ term dramatically increases $z_{\text {min }}$. However, the influence of the small cosmological constant may be ignored in any case.

At the end of our considerations, we propose another astrophysical test, which distinguishes string- and matter-dominated models more distinctly than the previous test. The test consists in counting galaxies with a given redshift. Denoting this number by $N(z)$ and coming back to the formula (68), we find that

$$
N(z)=4 \pi n(z) S_{k}^{2}[\chi(z)] \frac{d \chi}{d z},
$$

We shall not examine this function in detail. As in the case of the luminosity distance, we can expand it into series with respect to $z$ (we put for simplicity $n(z)=$ const. $=n$.

$$
\begin{aligned}
N(z) & =4 \pi n\left(\frac{4 \sigma_{r 0}+3 \sigma_{m 0}+2 \sigma_{s 0}-q_{0}-1}{k}\right)^{\frac{3}{2}} \\
& \times\left\{z^{2}-2\left(q_{0}+1\right) z^{3}+\frac{1}{12}\left[\left(q_{0}+1\right)\left(37 q_{0}+31\right)-96 \sigma_{r 0}-42 \sigma_{m 0}-8 \sigma_{s 0}\right] z^{4}+\ldots\right\} .
\end{aligned}
$$

In contradistinction to the previous case concerning $D_{0}$, now strings appear in each order of the expansion, in a constant factor. Coming back to the full formula (78), we notice that the function $N(z)$ possesses a maximum for some value $z_{\max }$. We are interested in only the dependence of $z_{\max }$ on the density of cosmic strings. In order to extract main features of this dependence, we assume that $\lambda=\sigma_{r 0}=k=0$. Then $\chi$ is given by Eq. (53). Differentiating $N(z)$ with respect to $z$ and equating the result to zero, we find the following formula for $z_{\max }$ :

$$
\begin{aligned}
\sinh \frac{2 \sqrt{2 \sigma_{s 0}\left[z\left(1-2 \sigma_{s 0}\right)+1\right]}}{(3 z+1)\left(\frac{1}{2}-\sigma_{s 0}\right)} & =\frac{\sqrt{2 \sigma_{s 0}}}{\left(\frac{1}{2}-\sigma_{s 0}\right)^{2}} \\
& \times \frac{z\left(\frac{1}{2}-\sigma_{s 0}\right)-\left(\sigma_{s 0}+\frac{1}{2}\right)\left(\sqrt{z\left(1-2 \sigma_{s 0}\right)+1}-1\right)}{z+1} .
\end{aligned}
$$

In two limiting models (matter- and string-dominated universes), the equation is solvable analytically, namely $z_{\max }=16 / 9$ for $\sigma_{s 0}=0$ and $z_{\max }=e^{2}-1$ for $\sigma_{m 0}=0$. Figure 3 shows the behaviour of $z_{\max }$ as a function of $\sigma_{s 0}$ in the whole allowed interval 
$\left(0, \frac{1}{2}\right)$. For comparison, we plot also, in the same coordinate system, the dependence of $z_{\min }$ on $\sigma_{s 0}$. From the diagram, we see that although for small density of strings the change of $z_{\max }$ (compared to the matter-dominated universe) is not remarkable, in the string-dominated model the shift of $z_{\max }$ is large. One may show that the influence of the cosmological constant $z_{\max }$ is qualitatively the same as in the case of $\theta_{\min }$.

\section{Conclusions}

The aim of the paper was to examine some observational implications of the universe filled with a sufficiently dense network of strings. The averaged energy density of strings was assumed to scale as $\varrho_{s} \sim R^{-2}$. Hence, we assumed the simplest possibility when the system of strings stretches conformally by the expansion and may come to dominate the universe. Besides cosmic strings, the universe contains non-interacting pressureless dust, radiation, and a cosmological constant. The mathematical formalism, which could describe such general models, is based on Weierstrass functions. We have given solutions of the Friedman equation and the principal astrophysical formulas for observable quantities in the models. The first conclusion from the results obtained was the confirmation of the general conviction that the influence of the cosmic strings on the evolution of the universe is relatively small in spite of the sophisticated equation of state for the strings (pressure given by strings is negative). On the basis of the derived formulas, we proposed some tests, which may be used for the verification, if not for the entire string scenario, at least for the string-domination scenario (of course, only in the case $\varrho_{s} \sim R^{-2}$. We showed that the luminosity distance and consequently the apparent-magnitude relations are not sensitive tests if one takes small accuracy of present observational data into account. In the next test, we examined the influence of the cosmic strings on the redshift $z_{\min }$, for which the angular size of galaxies takes a minimum value. We noticed that in the string-dominated universe $z_{\min }$ is shifted compared to the case without strings, and the value of this shift is of order $\frac{1}{2}$. We checked also that the small $\Lambda$ term (reasonable value for our present universe) does not change the result too much. The last test considered in the paper was connected with the source counts. We proved that the number of sources corresponding to a given redshift in a homogeneous, expanding universe possesses a maximum depending strongly on the strings. Strictly speaking, this dependence is not so distinct for a low energy density of strings. Then $z_{\max }$ is roughly equal to 2. However, in string-dominated universe, for example in the case when strings form invisible dark matter giving $\Omega=1, z_{\max }$ dramatically increases to $e^{2}-1$ for $\sigma_{s 0} \rightarrow \frac{1}{2}$, 
where $e=2.71828 \ldots$... From Fig.3 it follows that the string-dominated scenario (for example, where $90 \%$ of the total energy is in strings and the rest is in matter) should give $z_{\max }=4$. One should also emphasize that $z_{\max } \approx 2$ still allows existence of cosmic strings up to $40 \%$ of the total energy (in our considerations, we assumed that the number of sources in a comoving volume is constant in time). In spite of the strong dependence of $z_{\max }$ on the density of strings $\sigma_{s 0}$, practical application of the presented test does not seem to be possible up to now because of large values of redshifts which should be taken into account $(z>2)$. First, the problem of faint galaxies exists and there is a limit to how faint a galaxy one can detect. The second problem is even more severe and is connected with evolutionary effects of galaxies for large $z$. The full formula for this test should involve the appropriate corrections.

In conclusion, we would like to remind the reader that throughout the paper we considered only the simplest system of cosmic strings scaling as $\varrho_{s} \sim R^{-2}$. Discussion of observational implications of other, maybe more realistic, string-domination scenarios, where $\varrho_{s} \sim R^{-n}$ and $n>2$, will be published in the future.

\section{A. Astrophysical Formulas for the Cosmological Models with Domain Walls}

A redshift-magnitude relation for the universe which contains also the domain walls (with $\varrho_{w} \propto R^{-1}$ ) can be calculated following Dą̧browski and Stelmach (1988) ${ }^{1}$. Here we give the main result which generalizes our formula (47) which is a starting point for calculation all the observable quantities. Firstly, one introduces a new constant $C_{w}$ which is related to domain walls

$$
\varrho_{w} a=C_{w} \frac{3}{8 \pi G}
$$

and the dimensionless density parameter

$$
\sigma_{w 0}=\frac{4 \pi G \varrho_{w 0}}{3 H_{0}^{2}}
$$

\footnotetext{
${ }^{1}$ This appendix was not included in the original article, but for completness of the discussion of the whole spectrum of cosmic fluids with the equations of state from $p=-\varrho=-\Lambda$ to $p=\frac{1}{3} \varrho$ we include the domain walls following Dąbrowski, M.P., and Stelmach, J. ((1988). In Large Scale Structure in the Universe, IAU Symposium No. 130, edited by J. Audouze and A. Szalay (Reidel, Dordrecht)).
} 
The Friedman equation written down in terms of dimensionless variables 10) now takes the form

$$
\left(\frac{d T}{d \tau}\right)^{2}=\alpha T^{4}+\frac{2}{3} T^{3}-k^{\prime} T^{2}+\beta T+\frac{\lambda}{3},
$$

where

$$
\beta \equiv C_{w} \Lambda_{c}^{-1 / 2}
$$

The solution of the Eq. (A3)is now

$$
T(\tau)=\frac{3 \sqrt{\alpha} \mathcal{P}^{\prime}(\tau)-\mathcal{P}(\tau)-\frac{k^{\prime}}{12}-\frac{1}{48} \alpha \beta k^{\prime 2}}{6 \alpha \mathcal{P}(\tau)-\frac{1}{6}-k^{\prime} \alpha}
$$

where $\mathcal{P}$ is given by Eq. (16), i.e.,

$$
\mathcal{P}^{\prime}(\tau) \equiv \frac{d \mathcal{P}}{d \tau}=\sqrt{4 \mathcal{P}^{3}-g_{2} \mathcal{P}-g_{3}}
$$

and the invariants read as

$$
\begin{aligned}
& g_{2}=\frac{k^{\prime 2}}{12}+\frac{\alpha \lambda}{3}-\frac{\beta}{6} \\
& g_{3}=6^{-3}\left(k^{\prime 3}-2 \lambda-3 k^{\prime} \beta\right)-\frac{\alpha}{2}\left(\frac{k^{\prime} \lambda}{9}+\frac{\beta^{2}}{8}\right) .
\end{aligned}
$$

In the model parameters $(37),(38),(39),(40),(41)$ and (42) one has to replace $\left(4 \sigma_{r 0}+3 \sigma_{m 0}-q_{0}-1\right)$ by $\left(4 \sigma_{r 0}+3 \sigma_{m 0}+\sigma_{w 0}-q_{0}-1\right)$ and $\left(4 \sigma_{r 0}+3 \sigma_{m 0}+2 \sigma_{s 0}-q_{0}-1\right)$ by $\left(4 \sigma_{r 0}+3 \sigma_{m 0}+2 \sigma_{s 0}+\sigma_{w 0}-q_{0}-1\right)$. After that the formula (47) generalizes to

$$
\begin{aligned}
\mathcal{P}(\chi)=\frac{k}{4 \sigma_{r 0}+3 \sigma_{m 0}+2 \sigma_{s 0}+\sigma_{w 0}-q_{0}-1} \times \\
\left(\frac{4 \sigma_{r 0}+3 \sigma_{m 0} \sigma_{w 0}-q_{0}-1}{6}-\frac{z+2}{2}\left[\sigma_{m 0}+\sigma_{r 0}(z+2)\right]\right. \\
\left.+\frac{1}{4 z^{2}}\left\{1+\left[2 \sigma_{r 0} z^{2}(z+2)^{2}+\sigma_{m 0} z^{2}(2 z+3)-\sigma_{w 0} z^{2}+q_{0} z(z+2)+(z+1)^{2}\right]^{\frac{1}{2}}\right\}\right) .
\end{aligned}
$$

Inserting $\chi$ calculated as an inverse function of $\mathcal{P}$ from (A9) into (49), (67), and (68) allows to find a redshift-magnitude relation, angular diameter, and the number of galaxies with redshifts from $z$ to $z+d z$ in the universe which is filled with all the cosmological fluids: radiation with $p_{r}=\frac{1}{3} \varrho_{r}$, dust with $p_{m}=0$, cosmic strings with $p_{s}=-\frac{1}{3} \varrho_{s}$, domain walls with $p_{w}=\frac{2}{3} \varrho_{w}$, and the cosmological constant with $p_{\Lambda}=-\varrho \Lambda=-\Lambda$. 


\section{REFERENCES}

Abramovitz, M., and Stegun, I.A. (1964) Handbook of Mathematical Functions (Dover, New York).

Aryal, M., Everett, A.E., Vilenkin, A., and Vachaspati, T. (1986). Phys. Rev. D 34, 434.

Bennet, D.P. (1986a). Phys. Rev. D 33, 872.

Bennet, D.P. (1986b). Phys. Rev. D 34, 3592.

Charlton, J., and Turner, M. (1987). Astrophys. J. 313, 495.

Coquereaux, R., and Grossmann A. (1982). Ann. Phys. (N.Y.) 143, 296.

Dạbrowski M.P., and Stelmach J. (1986a). Ann. Phys. (N.Y.) 166, 422.

Dąbrowski M.P., and Stelmach J. (1986b). Astron.J. 92, 1272.

Dąbrowski M.P., and Stelmach J. (1987a). Astron.J. 94, 1373.

Dąbrowski M.P., and Stelmach J. (1987b). N. Copernicus Astron. Center Warsaw Prepr. No. 174.

Edwards, D. (1972). Mon. Not. R. Astron. Soc. 159, 51.

Ellis, G.F.R., and MacCallum, M.A.H. (1970). Commun. Math. Phys. 19, 31.

Gott, J.R. (1985). Astrophys. J. 288, 422.

Gott, J.R., and Rees, M.J. (1987). Mon. Not. R. Astron. Soc. 227, 453.

Guth, A.H. (1981), Phys. Rev. D 23, 347.

Hiscock, W.A. (1985). Phys. Rev. D 31, 3288.

Hogan, C.J., and Rees, M.J. (1984). Nature 311, 109.

Kaiser, N., and Stebbins, A. (1984). Nature 310, 391.

Kardashev, N.S. (1986). Sovi. Astron. 30, 498.

Kibble, T.W.B. (1976). J. Phys. A 9, 1387.

Kibble, T.W.B. (1975). Nucl. Phys. B 252, 227. 
Kibble, T.W.B. (1986a). In Particles and the Universe, edited by G. Lazarides and Q. Shafi (Elsevier, New York).

Kibble, T.W.B. (1986b). Phys. Rev. D 33, 328.

Kristian, J., and Sachs, R.K. (1966). Astrophys. J. 143, 379.

Lang, K.R. (1974). Astrophysical Formulas (Springer, Berlin).

Loh, E., and Spillar, E. (1986a). Astrophys. J. Lett. 307, L1.

Loh, E., and Spillar, E. (1986b). Astrophys. J. 303, 154.

Narlikar, J.V. (1983). Introduction to Cosmology (Jones and Bartlett, Boston).

Scherrer, R.J., and Frieman, J.A. (1986). Phys. Rev. D 33, 3556.

Solheim, J.E. (1966). Mon. Not. R. Astr. Soc. 133, 321.

Tricomi, F. (1937). Funzioni Ellittiche, edited by Nicola Zanichelli, Bologna.

Turok, N., and Bhattacharjee, P. (1984). Phys. Rev. D 29, 1557.

Turok, N., and Brandenberger, R.H. (1986). Phys. Rev. D 33, 2175.

Turner, M.S. (1985). Phys. Rev. Lett. 54, 252.

Turner, M.S. (1987). In Large scale Structure in the Universe, IAU Symposium No. 130, edited by J. Audouze and A. Szalay (Reidel, Dordrecht).

Vachaspati, T., and Vilenkin, A. (1984). Phys. Rev. D 30, 2036.

Vachaspati, T., and Vilenkin, A. (1987). Phys. Rev. D 35, 1311.

Vilenkin, A. (1981a). Phys. Rev. D 23, 852.

Vilenkin, A. (1981b). Phys. Rev. Lett. 46, 1169.

Vilenkin, A. (1981c). Phys. Rev. D 24, 2082.

Vilenkin, A., and Shafi, Q. (1983). Phys. Rev. Lett. 51, 1716.

Vilenkin, A. (1984a). Phys. Rev. Lett. 53, 1016.

Vilenkin, A. (1984b). Astrophys. J. Lett. 282, L51.

Vilenkin, A. (1985). Phys. Rep. 121, 265. 
Zel'dovich, Y.B., Kobzarev, I.Yu., and Okun, L.B. (1974). Zh. Eksp. Teor. Fiz. 67, 3 [(1975). Sov. Phys.-JETP 40, 1)].

Zel'dovich, Y.B. (1980). Mon. Not. R. Astron. Soc. 192, 663.

This preprint was prepared with the AAS IATEX macros v5.2. 


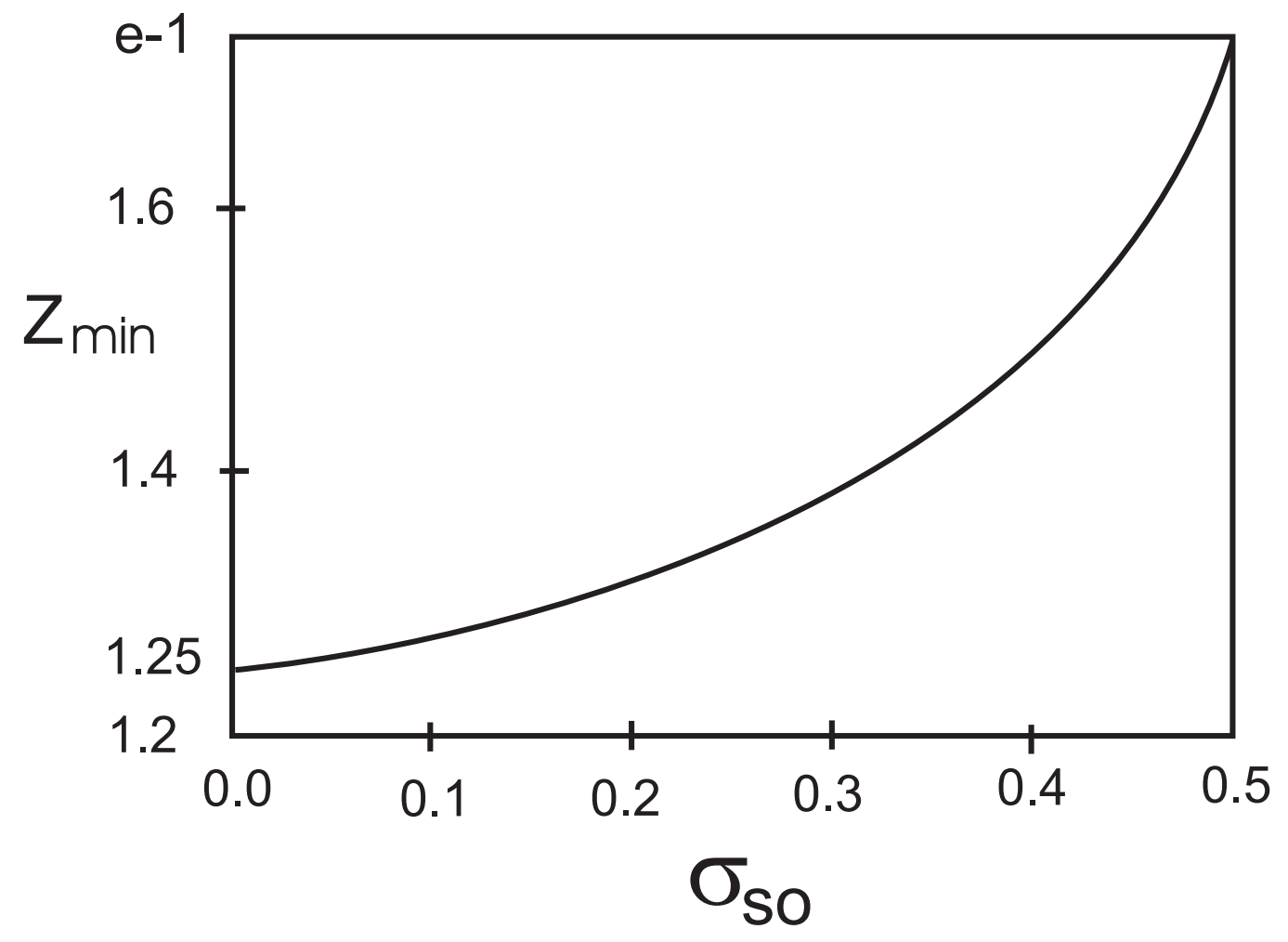

Fig. 1.- Dependence of the redshift, for which the regular size of a galaxy takes a minimum value, on the energy density of strings. $\lambda=k=\sigma_{s 0}=0, \sigma_{m 0}+\sigma_{s 0}=\frac{1}{2}$. 


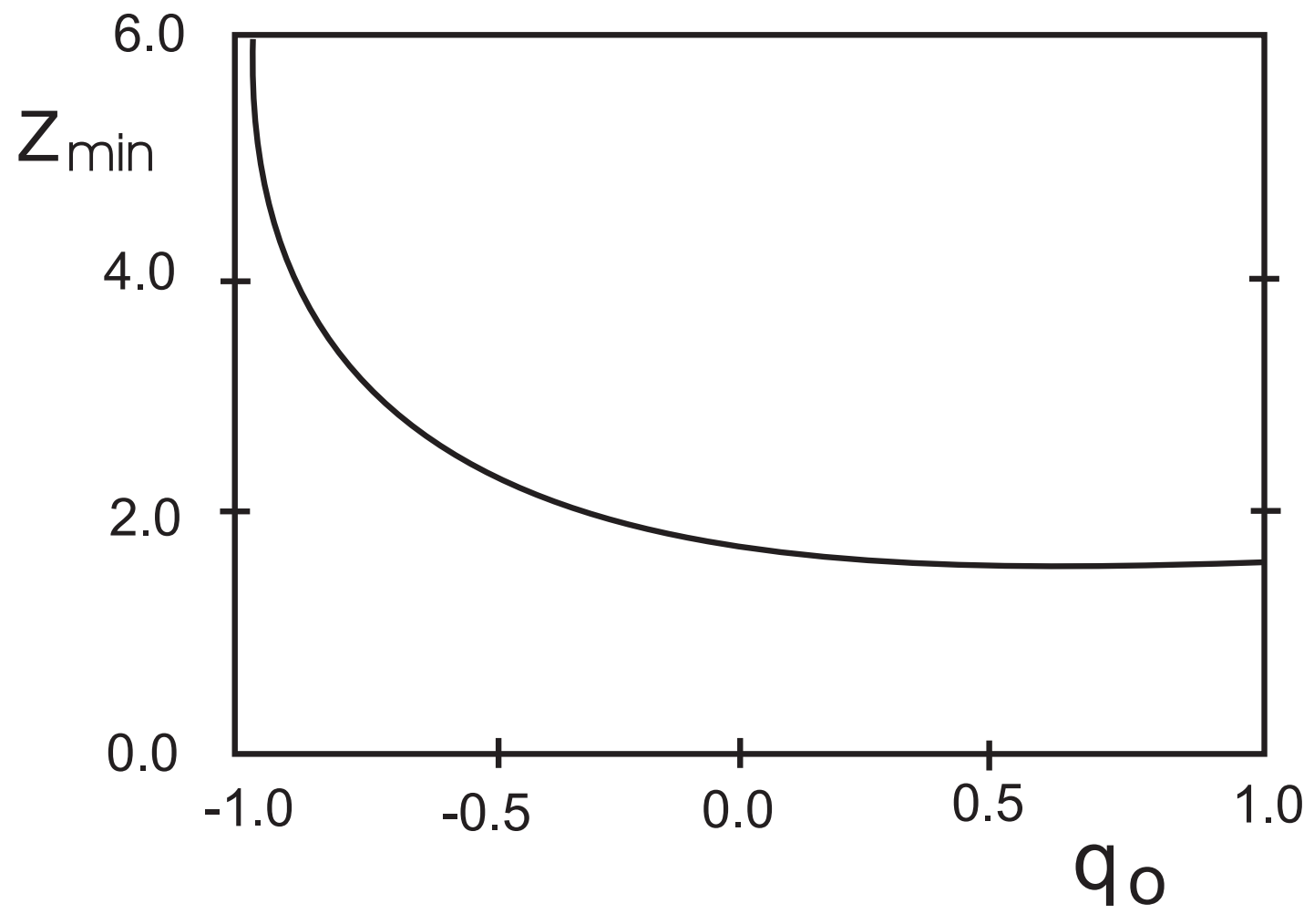

Fig. 2.- Influence of the cosmological constant on $z_{\min } . \sigma_{r 0}=\sigma_{m 0}=k=0, \sigma_{s 0}=\frac{1}{2}$. 


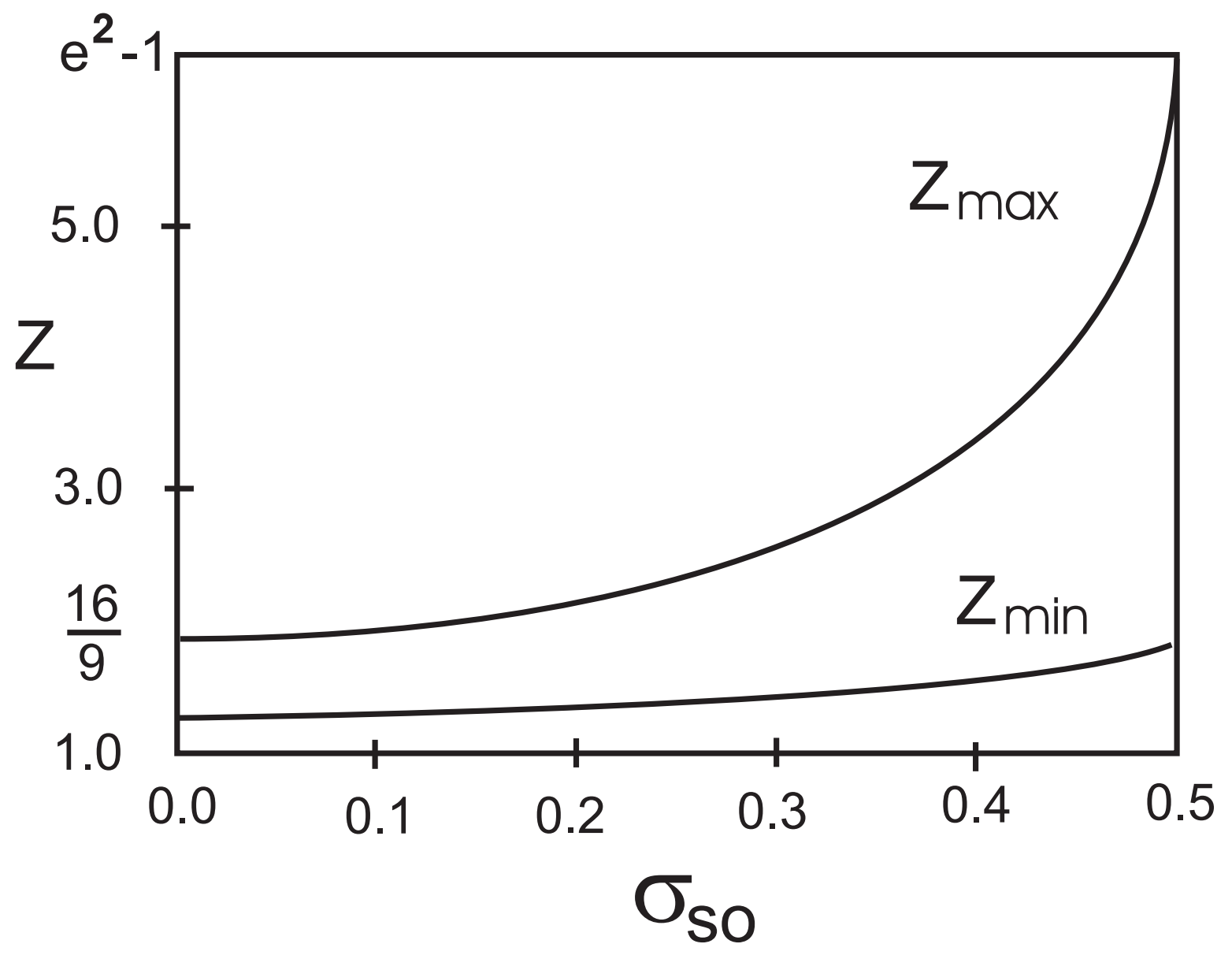

Fig. 3.- Dependence of the redshift, for which $N(z)$ takes a maximum value, on the energy density of strings. $\lambda=k=\sigma_{r 0}=0, \sigma_{m 0}+\sigma_{s 0}=\frac{1}{2}$. 